\title{
Uniform and Simultaneous Orthogonal Functionalization of a Metal-Organic Framework Material
}

\author{
Kanchana P. Samarakoon, ${ }^{a}$ Mohammad S. Yazdanparast, ${ }^{a}$ Victor W. Day ${ }^{\mathrm{b}}$ and Tendai Gadzikwa*a \\ aDepartment of Chemistry, Kansas State University, Manhattan, Kansas 66506, USA.

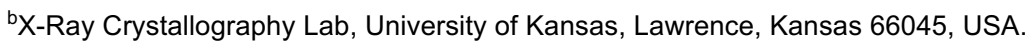 \\ *gadzikwa@ksu.edu
}

\begin{abstract}
We exploit orthogonal chemical reactivity to generate uniformly multifunctionalized confined spaces. A metal-organic framework (MOF) material that has azide and hydroxyl reactive groups in well-defined locations is independently functionalized to yield a uniformly bifunctionalized material via multiple paths, including via a simultaneous, one-pot reaction.
\end{abstract}

Of the conceptual pillars that underpin our strategies for the synthesis of complex molecular systems, orthogonality is perhaps the most powerful. Whether the multifunctional system under consideration is a small molecule or an extended structure, our ability to control the location of desired functional groups is often predicated on having an advanced synthetic intermediate that has orthogonally reactive sites. Thus, orthogonality is a crucial tool for synthetic chemists as we pursue the construction of highly functional materials that model the cooperative behaviour of biological systems. ${ }^{[1]}$

A variety of supramolecular systems have been assembled over the decades to mimic the rich host-guest chemistry found in Nature. ${ }^{[2-4]}$ Of those constructs, metal-organic framework (MOF) materials may be the most suited to the task. ${ }^{[5-7]}$ Their cavities are on the same length scale as small molecules and they can be tuned by a vast array of organic functionalities that are capable of supramolecular interactions. What MOFs lack, however, is the well-defined complexity that is integral to the function of many biological structures. To bridge this gap, MOF chemists have recently been developing methods to generate uniformly multifunctional MOFs ${ }^{[8-11]}$ uniformity being vital to achieving selectivity in whatever process the MOFs are engaged. ${ }^{[12,13]}$

Our recent contribution to this work is a strategy for the uniform bifunctionalization of MOF materials. ${ }^{[14]}$ We constructed a large-channel, pillared MOF, KSU-1, composed of two linkers that have distinct coordination chemistry and that bear independently reactive functionalities. The different coordination chemistry of the linkers guarantees that the independently reactive groups are in well-defined locations throughout the structure, ${ }^{[9,15]}$ resulting in uniform binary functionalization (Figure $1 \mathrm{~A}$ ). While this is an efficient methodology for generating confined spaces that are both complex and uniform, the system has a drawback that limits its general use: the amine and hydroxyl reactive groups of KSU1 both react as nucleophiles. While the hydroxyls' poorer aptitude for nucleophilic attack ${ }^{[16]}$ allows for independent reaction, the first functionalization is restricted to reacting the amines with the limited range of electrophiles that will not also react with the $\mathrm{OH}$ group. Additionally, the material cannot be modified with moieties that contain better nucleophiles than the hydroxyls. As biological function often involves the action of nucleophilic amines and thiols, ${ }^{[17]}$ this constraint severely diminishes our ability to construct biomimetic materials.
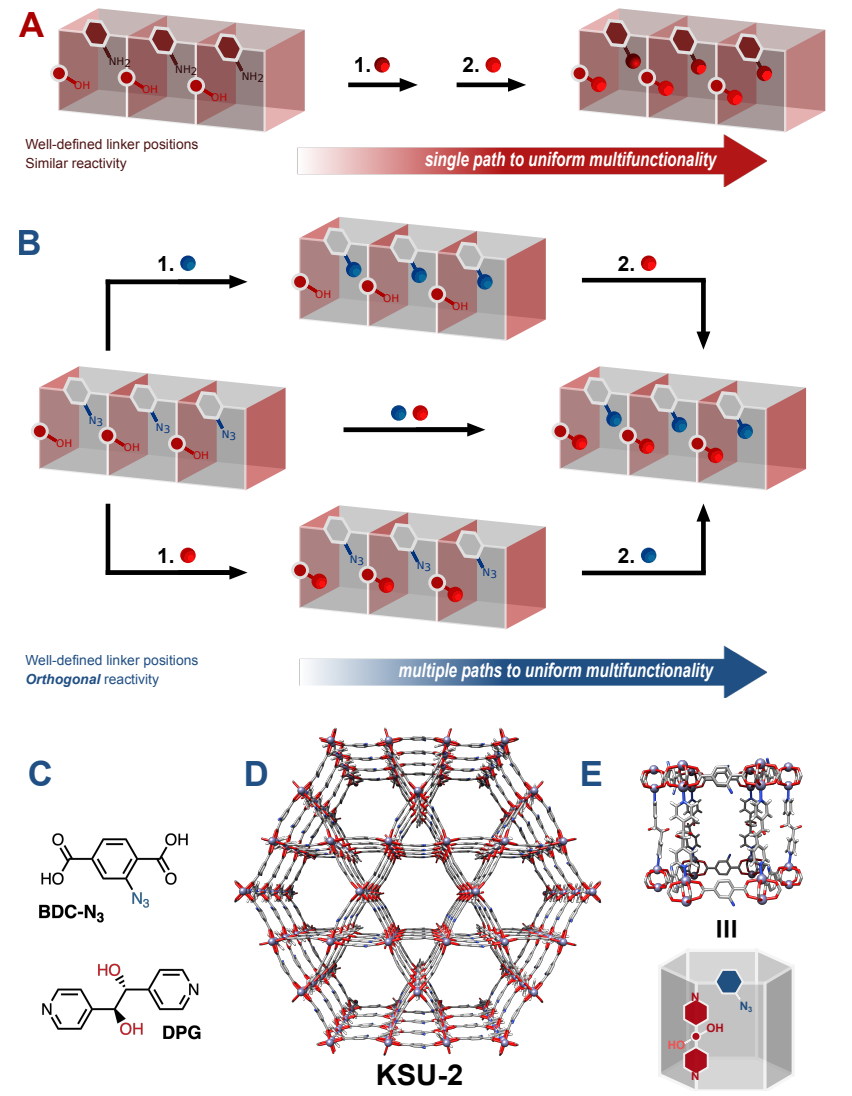

Figure 1. Functionalization of regularly positioned, independently reactive MOF linkers results in a uniformly multifunctional MOF. B: The use of orthogonally reactive linkers yields a uniformly multifunctional MOF via multiple routes. C: Orthogonally reactive linkers. D: KSU-2 viewed down the $c$-axis. E: Network unit of KSU-2 and its schematic representation.

To remedy these limitations, we have developed a system in which the modifiable components have the orthogonal reactivity. While the construction of MOFs with orthogonally reactive linkers has been reported, the use of identical coordination chemistry resulted in randomly distributed functionality. ${ }^{[18-20]}$ Our goal in this work is to demonstrate an orthogonal functionalization strategy that not only yields uniformly functionalized MOFs, but that can also do so via multiple paths, including via a simultaneous reaction (Figure 1B). Multipath reactions allow us to react the $\mathrm{OH}$ first, providing greater flexibility in reactant choice for the second reaction. Our approach is to construct a MOF with the Kagome-type topology of KSU-1 because its channels are large enough to accommodate multiple additional functional groups. The amine groups are replaced by azides which can be functionalized via the copper(I) catalyzed azide alkyne 
cycloaddition (CuAAC) reaction. ${ }^{[21-23]}$ Under CuAAC conditions, only azides react with terminal alkynes, and vice versa. While not entirely biorthogonal, the reaction is tolerant to the presence of the functional groups found in biology. ${ }^{[2]}$ Thus, this robust and selective reaction is favorable for the functionalization of materials that are already highly functionalized.

We undertook the construction of an orthogonally functionalizable MOF using 2-azido-1,4-benzenediacarboxylic acid (BDC-N 3 ) and meso- $\alpha, \beta$-di(4-pyridyl)glycol (DPG) as our organic linkers (Figure $1 \mathrm{C}$ ). The linkers were incubated with $\mathrm{Zn}\left(\mathrm{NO}_{3}\right)_{2} \cdot 6 \mathrm{H}_{2} \mathrm{O}$ in DMF at $60{ }^{\circ} \mathrm{C}$ for $24 \mathrm{~h}$, yielding yellow, blocklike crystals of KSU-2. Single-crystal X-ray diffraction analysis revealed the crystals to be those of a mixed-linker MOF material (Figure 1D-E). The BDC- $\mathrm{N}_{3}$ linkers are connected by $\mathrm{Zn}$ paddlewheel secondary building units (SBUs) in the ab-plane, forming 2D Kagome-type sheets that are pillared by DPG linkers along the $c$-axis. The triangular and hexagonal channels running down the $c$-axis are $1 \mathrm{~nm}$ and $1.7 \mathrm{~nm}$ across, respectively. The purity of bulk phases of KSU-2 was determined by powder X-ray diffraction (PXRD), Figure $\mathrm{S} 1$, and supported by ${ }^{1} \mathrm{H}-\mathrm{NMR}$ spectroscopy of the material digested in a solution of $\mathrm{D}_{2} \mathrm{SO}_{4}$ in DMSO- $d_{6}$ (Figures $2 \mathrm{~B}$ and $\mathrm{S} 2$ ). The material does not remain porous under evacuation, as determined by $\mathrm{N}_{2}$ adsorption (Figure S3), however, thermogravimetric analysis (TGA) of KSU-2 (Figure S4) indicates that the material has substantial solventaccessible volume. The MOF loses $\sim 45 \%$ of its weight as DMF upon heating, supporting our expectation that KSU-2 would have ample room for multifunctionalization.

For the multifunctionalization of KSU-2, we elected to react the azides with phenylacetylene, and the hydroxyls with isopropyl isocyanate (Figure 2A). Following a modified literature procedure, ${ }^{[25]} \mathrm{KSU}-2$ was incubated in a DMF solution of phenylacetylene and $\mathrm{CuBr}$ catalyst under a nitrogen atmosphere at $60^{\circ} \mathrm{C}$, with constant agitation. Monitoring the reaction product by observing the disappearance of the azide stretching frequency peak of KSU-2 in the Fourier-transform infrared (FTIR) spectrum (Figure S5) revealed that the reaction was complete after $72 \mathrm{~h}$. Additionally, the ${ }^{1} \mathrm{H}-\mathrm{NMR}$ spectrum of the digested material had peaks corresponding to protons of both DPG and the $B D C_{\text {triazole }}{ }^{[26]}$ and none for $\mathrm{BDC}-\mathrm{N}_{3}$ (Figure $2 \mathrm{~B}$ ). High resolution electrospray ionization mass spectrometry (ESI-MS) of a solution of KSU2 triazole disassembled in a solution of DABCO in DMSO gave a positive mode spectrum with mass signals corresponding to $\left[B C_{\text {triazole }}-\mathrm{H}^{+}\right]$and $\left[\mathrm{DPG}+\mathrm{H}^{+}\right]$(Figure S6), confirming the quantitative and selective grafting of the alkyne to the azide. Following confirmation of crystallinity by PXRD (Figure S1) and retention of significant solvent-accessible volume by TGA ( 30 weight \% solvent, Figure S4), we proceeded with the functionalization of the DPG ligand of KSU-2 triazole.
A
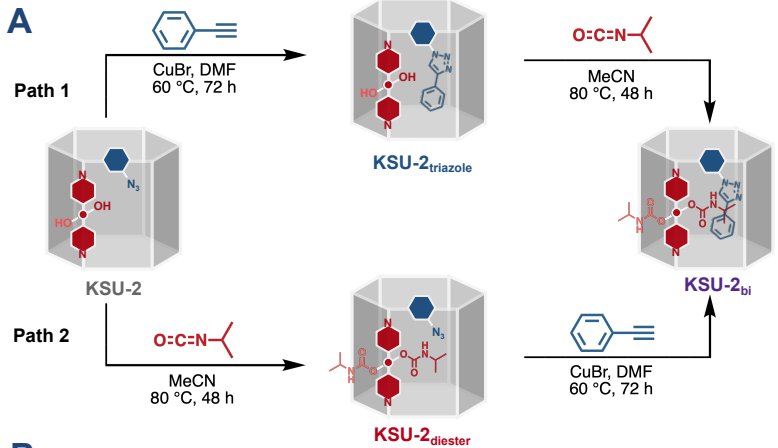

B

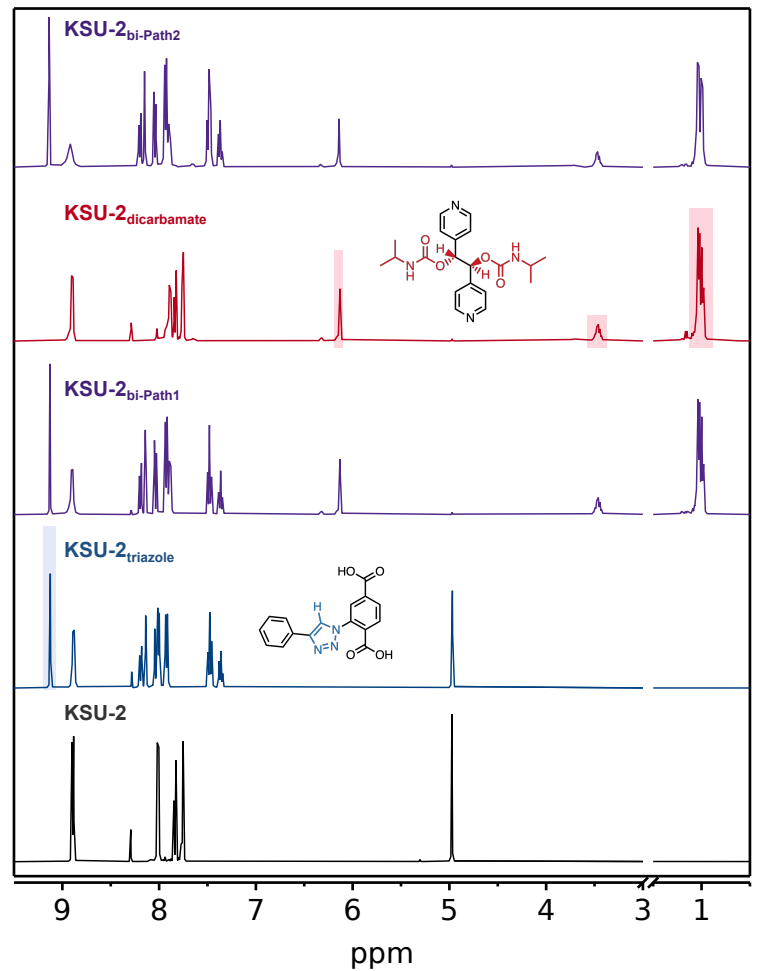

Figure 2. A: Schematic representation of the multipath, orthogonal functionalization of KSU-2 with phenyl acetylene and isopropyl isocyanate. B:

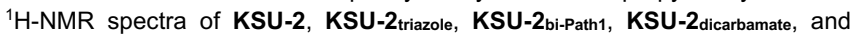
KSU-2 bi-Path2 digested in a solution of $\mathrm{D}_{2} \mathrm{SO}_{4}$ in DMSO-d6. C: FT-IR spectra of each material in the functionalization sequence, focusing on the triazole peak at $2117 \mathrm{~cm}^{-1}$. B: Simultaneous, independent functionalization of KSU-2 with phenyl acetylene and isopropyl isocyanate.

Isocyanates have been used before to functionalize aminebearing MOFs, ${ }^{[27-29]}$ and we selected the same electrophile to react with the hydroxyls of DPG to produce DPG dicarbamate. Previously we reacted DPG with a cyclic anhydride resulting in products with ester linkages. ${ }^{[14]}$ The advantage of the current reaction is that the carbamate linkage has higher chemical stability, ${ }^{[30]}$ which simplifies characterization of the products. Following a modified procedure, ${ }^{[31]}$ we immersed $\mathbf{K S U}-2$ triazole in a solution of isopropyl isocyanate in acetonitrile, with continuous agitation at $80{ }^{\circ} \mathrm{C}$ for $48 \mathrm{~h}$ to produce $\mathbf{K S U}-\mathbf{2}_{\text {bi-Path1. The complete }}$ shift of the protons alpha to the glycol in the ${ }^{1} \mathrm{H}-\mathrm{NMR}$ spectrum of

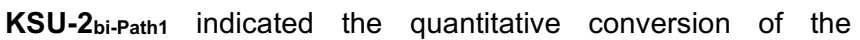
hydroxyls to carbamates (Figure 2B). The covalent grafting of the isocyanate to the hydroxyls was confirmed by disassembling KSU-2 bi-Path1 $_{1}$ in a solution of DABCO in DMSO- $d_{6}$. High resolution electrospray ionization mass spectrometry (ESI-MS) of the 
solution gave a positive mode spectrum that pseudo molecular ion signals corresponding to $\left[\mathrm{BDC}_{\text {triazole }}-\mathrm{H}^{+}\right]$and $\left[D \mathrm{PG}_{\text {dicarbamate }}+\mathrm{H}^{+}\right]$. The most significant peaks corresponded to the McLafferty rearrangement fragment $\left[D P G_{\text {dicarbamate }}-\mathrm{CO}_{2}-\right.$ $\left.{ }^{i} \mathrm{PrNH}_{2}+\mathrm{H}^{+}\right],{ }^{[32,33]}$ as well as that fragment ion minus neutral iPrNCO. ${ }^{[34]}$ The PXRD pattern (Figure S1) indicates that the material retains its crystallinity and the TGA confirms that an appreciable amount of solvent-accessible volume remains, as is indicated by the $\sim 20 \%$ weight loss of DMF (Figure S4).

Having established that the independent binary functionalization of KSU-2 to produce $\mathbf{K S U}-\mathbf{2}_{\text {bi-Path1 }}$ was possible via Path 1 , we then attempted to obtain the same product via Path 2 (Figure 2A). Using our previous conditions (vide supra) we first performed the carbamate formation reaction with KSU-2 to yield

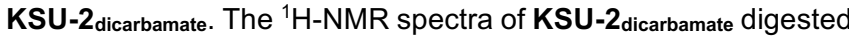
in acid (Figure 2B) confirmed the covalent attachment of isocyanate to DPG, as did the ESI-MS of the DABCO-digested solution (Figure S6). Additionally, a peak corresponding to the azide stretch of BDC- $\mathrm{N}_{3}$ was still present in the FTIR (Figure S5). The CUAAC reaction of $\mathbf{K S U}-\mathbf{2}_{\text {dicarbamate }}$ with phenylacetylene produced a material, KSU-2 $\mathbf{b i - P a t h 2 , ~ w i t h ~ t h e ~ s a m e ~ c h a r a c t e r i s t i c s ~}$

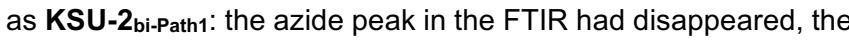
NMR had peaks corresponding to BDC triazole, and the ESI-MS had signals for $\left[D P G_{\text {dicarbamate }}+\mathrm{H}^{+}\right]$and $\left[\mathrm{BDC}_{\text {triazole }}+\mathrm{H}^{+}\right]$(Figure S6).
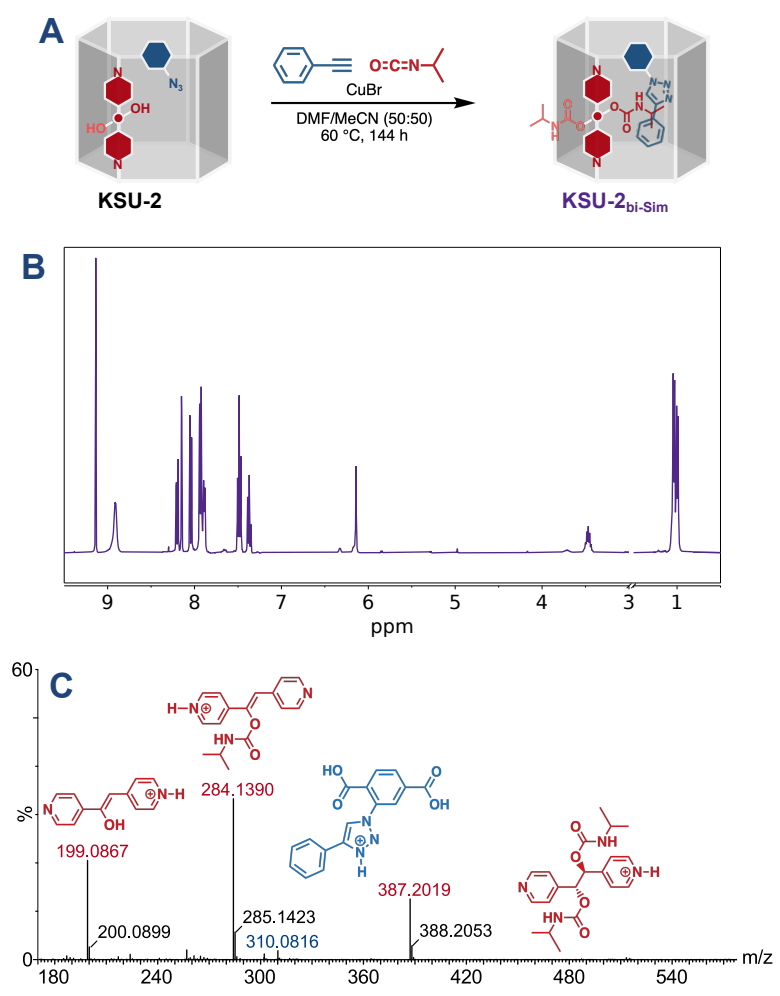

Figure 3. A: Schematic representation of the simultaneous, orthogonal functionalization of KSU-2 with phenyl acetylene and isopropyl isocyanate. B: ${ }^{1} \mathrm{H}-\mathrm{NMR}$ spectra of the simultaneous reaction product, $\mathrm{KSU}-\mathbf{2}$ bi-Sim digested in a solution of $\mathrm{D}_{2} \mathrm{SO}_{4}$ in DMSO- $d_{6}$. C: High resolution, positive mode, mass

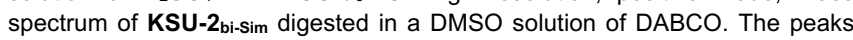
correspond to $\left[D P G_{\text {dicarbamate }}+\mathrm{H}^{+}\right.$], along with its McLafferty rearrangement fragments, and $\left[B D C_{\text {triazole }}-\mathrm{H}^{+}\right]$
To demonstrate an additional advantage of orthogonal reactivity, we sought to obtain the same final product by performing the functionalizations in a simultaneous reaction (Figure 3A). Using a DMF:MeCN mixture (50:50, v/v), KSU-2 was incubated in a solution of isopropyl isocyanate, phenylacetylene, and $\mathrm{CuBr}$ catalyst under a nitrogen atmosphere at $60{ }^{\circ} \mathrm{C}$, with constant agitation. Characterization of the final product after six days confirmed the success of the one pot, independent reaction. The ${ }^{1} \mathrm{H}-\mathrm{NMR}$ (Figure 3B), ESI-MS (Figures $3 \mathrm{C}$ and S6) and FTIR spectra (Figure S5) were all consistent with the formation of the bifunctional material KSU-2bi-Sim.

In conclusion, we have improved the utility of our recently developed method for the uniform binary functionalization of MOFs. The orthogonal reactivity offers more flexibility in how the functionalizations can be undertaken. Of particular interest is that the azide group of KSU-2 is near bioorthogonal, meaning that the MOF can be functionalized with functionalities commonly found in biology without the need for sterically demanding protecting groups that may require harsh conditions to remove. Thus, this development propels us further in our quest to develop a general platform for generating confined spaces that are uniformly decorated with multiple functional groups, i.e. spaces that can imitate the cooperative functionality of those found in natural systems.

CCDC 1970367 contains the supplementary crystallographic data for this paper. These data are provided free of charge by The Cambridge Crystallographic Data Centre.

\section{Acknowledgements}

This study was supported by a National Science Foundation grant, CHE-1800517, and NSF-MRI grants, CHE-0923449 to the University of Kansas to purchase the X-ray diffractometer and software used in this study, and CHE-1826982 to Kansas State University for the NMR spectrometer used in this study. The authors also acknowledge the Aakeröy Lab at KState for use of their TGA.

Keywords: metal-organic frameworks • orthogonal functionalization • post-synthetic modification • supramolecular chemistry

[1] C.-H. Wong, S. C. Zimmerman, Chem. Commun. 2013, 49, 1679-

1695. Chem. Soc. Rev. 2014, 43, 1734-1787.

[3] S. Zarra, D. M. Wood, D. A. Roberts, J. R. Nitschke, Chem. Soc. Rev. 2014, 44, 419-432.

[4] E. Kuah, S. Toh, J. Yee, Q. Ma, Z. Gao, Chem. - Eur. J. 2016, 22, $8404-8430$

[5] M. Zhang, Z.-Y. Gu, M. Bosch, Z. Perry, H.-C. Zhou, Coord. Chem Rev. 2015, 293-294, 327-356.

[6] S. M. Cohen, Z. Zhang, J. A. Boissonnault, Inorg. Chem. 2016, 55, 7281-7290.

[7] I. Nath, J. Chakraborty, F. Verpoort, Chem. Soc. Rev. 2016, 45, 4127-4170.

[8] S. Yuan, Y.-P. Chen, J.-S. Qin, W. Lu, L. Zou, Q. Zhang, X. Wang,

X. Sun, H.-C. Zhou, J. Am. Chem. Soc. 2016, 138, 8912-8919.

[9] J.-S. Qin, S. Yuan, Q. Wang, A. Alsalme, H.-C. Zhou, J. Mater. Chem. A 2017, 5, 4280-4291.

[10] S. Pullen, G. H. Clever, Acc. Chem. Res. 2018, 51, 3052-3064.

[11] B. Tu, L. Diestel, Z.-L. Shi, W. R. L. N. Bandara, Y. Chen, W. Lin,

Y.-B. Zhang, S. G. Telfer, Q. Li, Angew. Chem. Int. Ed. 2019, 58, 5348-5353.

[12] 13936-13943.
L. Liu, T.-Y. Zhou, S. G. Telfer, J. Am. Chem. Soc. 2017, 139, 
[13] T.-Y. Zhou, B. Auer, S. J. Lee, S. G. Telfer, J. Am. Chem. Soc 2019, 141, 1577-1582

[14] K. P. Samarakoon, C. S. Satterfield, M. C. McCoy, D. A. PivaralUrbina, T. Islamoglu, V. W. Day, T. Gadzikwa, Inorg. Chem. 2019, 58, 8906 8909. X.-L. Luo, Z. Yin, M.-H. Zeng, M. Kurmoo, Inorg. Chem. Front. 2016, 3, 1208-1226.

[16] H. Mayr, A. R. Ofial, J. Phys. Org. Chem. 2008, 21, 584-595.

[17] E. M. Sletten, C. R. Bertozzi, Acc. Chem. Res. 2011, 44, 666-676.

2011, 47, 7629-7631.

[19] C. Liu, T.-Y. Luo, E. S. Feura, C. Zhang, N. L. Rosi, J. Am. Chem. Soc. 2015, 137, 10508-10511.

[20] U. Fluch, B. D. McCarthy, S. Ott, Dalton Trans. 2018, 48, 45-49.

[21] C. W. Tornøe, C. Christensen, M. Meldal, J. Org. Chem. 2002, 67, 3057-3064.

[22] V. V. Rostovtsev, L. G. Green, V. V. Fokin, K. B. Sharpless,

Angew. Chem. Int. Ed. 2002, 41, 2596-2599.

[23] J. E. Hein, V. V. Fokin, Chem. Soc. Rev. 2010, 39, 1302-1315.
[24] 6974-6998.

[25] X. C. Yi, F -G. Xi, Y Qi, E - . Gao, RSC Adv. 2014, 5, 893-900. [26] M. Savonnet, D. Bazer-Bachi, N. Bats, J. Perez-Pellitero, E. Jeanneau, V. Lecocq, C. Pinel, D. Farrusseng, J. Am. Chem. Soc. 2010, 132, 4518-4519.

[27] E. Dugan, Z. Wang, M. Okamura, A. Medina, S. M. Cohen, Chem Commun. 2008, 3366-3368.

[28] S. J. Garibay, Z. Wang, K. K. Tanabe, S. M. Cohen, Inorg. Chem. 2009, 48, 7341-7349.

[29] S. Bernt, V. Guillerm, C. Serre, N. Stock, Chem. Commun. 2011 $47,2838-2840$.

[30] A. K. Ghosh, M. Brindisi, J. Med. Chem. 2015, 58, 2895-2940.

[31] X.-W. Dong, T. Liu, Y.-Z. Hu, X.-Y. Liu, C.-M. Che, Chem.

Commun. 2013, 49, 7681-7683.

[32] F. W. McLafferty, Anal. Chem. 1959, 31, 82-87.

[33] C. Wolf, C. N. Villalobos, P. G. Cummings, S. Kennedy-Gabb, M.

A. Olsen, G. Trescher, J. Am. Soc. Mass Spectrom. 2005, 16, 553-564.

[34] Y. Zhou, J. Guan, W. Gao, S. Lv, M. Ge, Molecules 2018, 23, 2496. 